\title{
Oxidant/antioxidant status, paraoxonase activity, and lipid profile in plasma of ovariectomized rats under the influence of estrogen, estrogen combined with progesterone, and genistein
}

\author{
This article was published in the following Dove Press journal: \\ Drug Design, Development and Therapy \\ 10 June 2015 \\ Number of times this article has been viewed
}

\author{
Elif Agacayak' \\ Serdar Basaranoglu \\ Senem Yaman Tunc' \\ Mehmet Sait Icen' \\ Fatih Mehmet Findik' \\ Ibrahim Kaplan ${ }^{3}$ \\ Osman Evliyaoglu ${ }^{3}$ \\ Talip Gul' \\ 'Department of Obstetrics and \\ Gynecology, Dicle University School \\ of Medicine, Diyarbakir, Turkey; \\ ${ }^{2}$ Department of Obstetrics and \\ Gynecology, Idil State Hospital, Sirnak, \\ Turkey; ${ }^{3}$ Department of Biochemistry, \\ Dicle University School of Medicine, \\ Diyarbakir, Turkey
}

Introduction: The aim of this study was to investigate whether estradiol (E2), E2 combined with progesterone (Prog) (E2/Prog), and genistein (Gen) treatment had antioxidative and antihyperlipidemic effects in the plasma of ovariectomized (OVX) rats.

Materials and methods: Adult female Sprague-Dawley rats were divided into five groups. Rats in all groups, except for those in a sham group, underwent bilateral ovariectomy under general anesthesia. The groups were as follows: sham group; control OVX group; group treated with estrogen $(0.014 \mathrm{mg} / \mathrm{kg} 17-\beta$ E2); group treated with a combination of E2 and Prog $(0.014 \mathrm{mg} / \mathrm{kg} 17-\beta$ E2 plus $0.028 \mathrm{mg} / \mathrm{kg}$ drospirenone), and group treated with Gen (10 mg/ $\mathrm{kg}$ /day). Plasma of rats of each treatment group was analyzed to determine the total antioxidant status, total oxidant status, paraoxonase activity, lipid profile, high-density lipoprotein (HDL-chol), low-density lipoprotein (LDL-chol), total cholesterol (Total-C), triacylglycerols, lipoprotein (a), and oxidative stress index.

Results: Plasma Total-C levels and body weight increased in all the OVX groups compared with the sham group $(P<0.005)$. The group treated with E2 had significantly elevated total oxidant status, oxidative stress index, LDL-chol, and Total-C compared with the control group $(P<0.005)$. Gen treatment might lead to lower LDL-chol and Total-C levels compared with E2 treatment.

Conclusions: Gen treatment might be preferred to E2 treatment for treatment of menopausal symptoms in patients at risk for cardiovascular diseases. However, considering the small sample size of this study, larger studies are needed in this area.

Keywords: genistein, menopause, cardiovascular diseases

\section{Introduction}

Vasomotor symptoms and genitourinary atrophy might develop in 75\%-80\% of women in the early menopausal period, and women might also suffer from osteoporosis and atherosclerotic cardiovascular diseases that might be life-threatening in the late period. ${ }^{1}$ Hormone replacement therapy (HRT) is used to relieve the menopausal symptoms. Increasingly, low-dose HRT and selective estrogen receptor modulators (SERMs) have been used during menopausal and postmenopausal periods. Genistein (Gen) is referred to as a phytoestrogen, an estrogen-like chemical compound present in some plants. There are two main types of phytoestrogens, namely isoflavones and lignans. The highest amounts of isoflavones are present in soy, and the most abundant isoflavone in soy is Gen. Gen is also abundant in red clover. ${ }^{2}$
Correspondence: Elif Agacayak

Department of Obstetrics and

Gynecology, Dicle University School

of Medicine, 21280, Diyarbakir, Turkey

Tel +905059433449

Fax +90 4I2 2488523

Email drelifagacayak@gmail.com 
The incidence of cardiovascular diseases is known to increase at menopause. ${ }^{3}$ However, women are not prescribed estrogen therapy during the menopausal period for prophylactic purposes against cardiac diseases. As a consequence, studies are still investigating alternative options to the estrogen therapy for patients at risk for cardiovascular diseases that might occur at menopause.

Lipoproteins, plasma triacylglycerol (TAG), and global high-molecular-weight cholesterol ester particles are the risk factors for cardiovascular diseases, especially for atherosclerosis. The density of lipoprotein molecule depends on the amount of cholesterol and triglyceride binding to the protein. ${ }^{4}$ Lipoprotein (a) has been found to be a new independent factor associated with cardiovascular diseases. ${ }^{5}$ Lipoprotein (a) is similar to low-density lipoprotein (LDL-chol); however, it has a lipoprotein (a) plasminogen-like structure due to the disulfide bridge. High-density lipoprotein (HDL-chol) provides protection against oxidative damage, thus playing a major role. The enzyme paraoxonase (PON1) associated with the HDL-chol surface modulates the antioxidant and anti-inflammatory role of HDL-chol. ${ }^{5}$

It has been shown that pronounced reduction in E2 leads to an increase in oxidative stress in the body, depending on the concentration and chemical structure of this hormone. Specifically, E2 at high concentrations inhibits the 8-hydroxylation of guanine bases of DNA. Thus it potentially has a beneficial antioxidant effect at high concentrations. However, disorders in genetic structure and DNA formation bring out the prooxidant effect of estrogen. In addition, glutathione-4-hydroxynonenal and malonaldehyde, which increase in the postmenopausal period, also have prooxidant effects just like estrogen at low concentrations. ${ }^{6}$ Furthermore, it has been shown that postmenopausal women have higher serum concentrations of inflammatory cytokines and prooxidant biomarkers, such as glutathione, 4-hydroxynonenal, and malonaldehyde, compared with premenopausal women. Elevated cytokines and prooxidant markers might be an indicator of oxidative stress in the postmenopausal period. ${ }^{7,8}$

In premenopausal women, the primary estrogen in circulation is $17-\beta$ E2. 17- $\beta$ E2 is a natural human estrogen and compensates for the physiological estrogen deficiency in the body, without affecting the liver functions. ${ }^{9}$ It has been shown that drospirenone has antimineralocorticoid and antiandrogenic effects. It combats the estrogen-induced stimulation of the renin-angiotensin-aldosterone system and stops testosterone from binding to androgen receptors. ${ }^{10}$

The current studies investigate phytoestrogens as an alternative option to synthetic estrogen, which might lead to potential complications. Like the other phytoestrogens, Gen either increases or decreases the effects of estrogen as it binds to the estrogen receptors (ERs: ER- $\beta$ and ER- $\alpha$ ) on cells. Gen binds to ER- $\beta 20$ times more frequently than to ER- $\alpha$. Gen stimulates both of these receptors; however, stimulation by Gen is not as powerful as the stimulation by real endogenous. In addition, it stops the attachment of the estrogen itself. Estrogen has been associated with risks for various cancers. For that reason, if Gen is regularly used by premenopausal women, it might help reduce that risk. On the other hand, Gen can partly compensate for human estrogen deficiency, such as after menopause. This is one of the reasons why Gen should be used to treat menopausal symptoms and prevent osteoporosis. ${ }^{11}$ Gen may be of help in prevention of heart diseases ${ }^{11,12}$ and in prevention and treatment of osteoporosis, ${ }^{13}$ as demonstrated by double-blind, placebo-controlled studies. Furthermore, Gen may help improve blood glucose control in people with prediabetes. ${ }^{14}$ Studies suggest potential benefits of Gen in cancer prevention, ${ }^{15,16}$ as well as in treatment of cancer ${ }^{16}$ and amyotrophic lateral sclerosis $;{ }^{17}$ however, these studies are based on low-level evidence.

The benefit of treatment with E2 alone vs E2 combined with progesterone (Prog), as well as the associated hyperlipidemic effects (including effects on atherosclerosis) and cardiovascular risks, are still debated. The aim of this study was to investigate whether E2 treatment, combined E2 plus Prog (E2/Prog) treatment, and Gen treatment have antioxidative and antihyperlipidemic effects on the plasma of ovariectomized (OVX) rats, and to investigate the effect of the phytoestrogen Gen on blood lipid profile and oxidative stress markers, as a potential alternative to HRT.

\section{Materials and methods}

\section{Animals, care, and nutrition}

This study was designed in accordance with the Animal Welfare Act and Guide for Care and Use of Laboratory Animals. It was carried out at Dicle University Health Sciences Practice and Research Center after the approval of Dicle University Local Ethics Committee for Care and Use of Laboratory Animals (approval number 2012/07) was obtained. A total of 50 adult female Sprague-Dawley rats, weighing between 250 and $300 \mathrm{~g}$, were kept in separate cages in the animal laboratory of Dicle University. They were maintained in a 12-hour light-dark cycle at room temperature $\left(25^{\circ} \mathrm{C} \pm 3^{\circ} \mathrm{C}\right)$ and were provided with standard rat chow and water ad libitum. All efforts were made to minimize animal suffering and to use the minimum number of animals necessary to produce reliable scientific data. 


\section{Experimental treatment}

Rats were randomized into five groups, with ten rats in each group, as described below:

Group 1 (sham group): The same surgical procedure was performed in the sham-operated rats except that the ovaries were not removed. Each rat received water without any medication, by orogastric catheter, once a day for 8 weeks.

Group 2 (control group): OVX rats received water without any medication, by orogastric catheter, once a day for 8 weeks.

Group 3: OVX rats received $10 \mathrm{mg} / \mathrm{kg} \mathrm{Gen}^{18}$ (Bonistein $\mathrm{S}^{\circledR}$; DSM Nutritional Products, Istanbul, Turkey), by orogastric catheter, once a day for 8 weeks.

Group 4: OVX rats received $0.014 \mathrm{mg} / \mathrm{kg} 17-\beta$ E2 ${ }^{18}$ (Estrofem $^{\circledR}$; Novo Nordisk, Bagsværd, Denmark), by orogastric catheter, once a day for 8 weeks.

Group 5: OVX rats received $0.014 \mathrm{mg} / \mathrm{kg} 17-\beta$ E2 plus $0.028 \mathrm{mg} / \mathrm{kg}$ drospirenone (Angeliq ${ }^{\circledR}$; Shering Alman), by orogastric catheter, once a day for 8 weeks.

In this study, drospirenone was used because it is a natural Prog-like progestin. Drospirenone is an analog of spironolactone. Unlike other progestogens, it has biochemical and pharmacologic profiles almost the same as endogenous
Prog, particularly regarding antimineralocorticoid and antiandrogenic activities. ${ }^{10}$

Ketamine hydrochloride $(100 \mathrm{mg} / \mathrm{kg}$, by intraperitoneal [IP] route) (Ketalar ${ }^{\circledR}$; Pfizer, Inc., New York, NY, USA) and xylazine $(1 \mathrm{mg} / \mathrm{kg} / \mathrm{IP})$ (Rompun ${ }^{\circledR}$; Bayer Healthcare AG, Leverkusen, Germany) were used to induce anesthesia. A midline abdominal incision was made at the pelvic level, under general anesthesia, in all groups. Bilateral ovariectomy was performed in all groups except for the sham group (Figure 1). Musculoperitoneum and fascia were sutured with 4/0 polyglactin (Ethicon ${ }^{\circledR}$; Ethicon, Inc., Somerville, NJ, USA), whereas the skin incision was sutured with $3 / 0$ atraumatic silk sutures. No antibiotics were administered before, during, or after the intervention, as effects and bioavailability of phytoestrogens depend on the route of administration, dosing, individual metabolism, administration of other pharmacologic agents, target tissue, concentration, number and type of ER, and presence or absence of endogenous estrogen. ${ }^{19}$

All treatments were initiated precisely 1 week after surgery. ${ }^{20}$ At the end of the study, all rats were sacrificed by intracardiac blood aspiration. In OVX rats, uterine atrophy was verified as an indicator of successful removal of both ovaries.
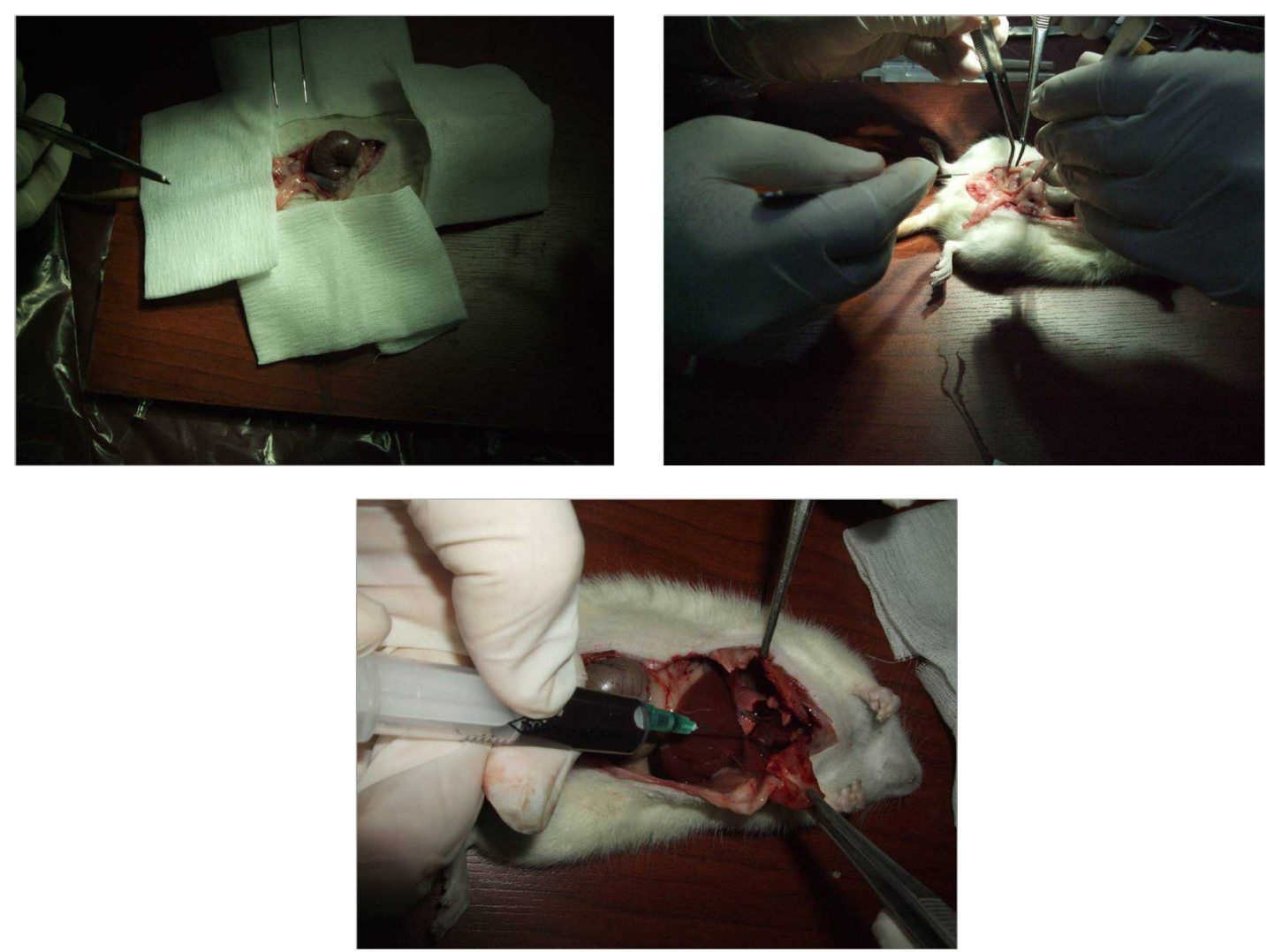

Figure I Bilateral ovariectomy performed after midline laparotomy. 


\section{Administration of genistein and estrogen}

For oral administration, E2 and Gen were dissolved in dimethyl sulfoxide (DMSO) 4\% (Sigma-Aldrich Corp, St Louis, MO, USA) (up to a concentration of $100 \mathrm{mM}$ ) and given by gavage. ${ }^{20,21}$

\section{Biochemical measurements}

In this study, the total antioxidant status (TAS) of supernatant fractions was assessed using a new automated and colorimetric measurement method that was developed by Erel. ${ }^{22}$ This is a 2,2V-azinobis-(3-ethylbenzothiazoline-6-sulfonate) (ABTSS+)-based method. In this method, the reduced form, ABTS, is oxidized to a typical blue-green ABTSS+. When the colored ABTSS+ is mixed with any oxidizable substance, it is reduced back to its original colorless ABTS form, where the reacted substance is oxidized. This is the fundamental principle of the methods that employ ABTS. The reduced ABTS is oxidized to ABTSS+ by means of hydrogen peroxide $\left(\mathrm{H}_{2} \mathrm{O}_{2}\right)$ alone, in the acidic medium. The concentrated ABTSS+ molecules remain stable for a long while in the acetate buffer solution. When diluted with a more concentrated acetate buffer solution at high $\mathrm{pH}$ values, the color is spontaneously and gradually bleached. Antioxidants in the sample speed up the bleaching rate to an extent proportional to their concentrations. This reaction can be viewed spectrophotometrically, and there is an inverse relation between the bleaching rate and the TAS of the sample. The reaction rate is calibrated with Trolox, which is a traditional standard for TAS measurements. The assay has excellent precision values, which are below $3 \%$. The TAS results are expressed as $\mu \mathrm{mol}$ Trolox equivalent/L.

The total oxidant status (TOS) of supernatant fractions was assessed using a new automated and colorimetric measurement method that was developed by Erel. ${ }^{23}$ Oxidants in the sample oxidize the ferrous ion-o-dianisidine complex to ferric ion. Glycerol molecules, which are abundant in the reaction medium, increase the oxidation reaction. The ferric ion gives a colored complex with xylenol orange, in an acidic medium. The color intensity can be assessed spectrophotometrically and is related to the total amount of oxidant molecules in the sample. The assay is calibrated with $\mathrm{H}_{2} \mathrm{O}_{2}$. The TOS results are expressed as mmol $\mathrm{H}_{2} \mathrm{O}_{2}$ equivalent/L.

Photometric measurements for total cholesterol (Total-C), TAG, HDL-chol, and LDL-chol were performed on an Abbott C 16000 device (Abbott Laboratories, Abbott Park, IL, USA). Lipoprotein (a) was determined by nephelometric method on a Beckman Immage ${ }^{\circledR} 800$ device (Beckman Coulter Inc, Brea, CA, USA). Serum PON1 activity was determined spectrophotometrically by means of modified Eckerson method. PON1 activity was detected using paraoxone as a substrate, with absorbance of 4-nitrophenol at $412 \mathrm{~nm} .{ }^{24}$ As a marker of the degree of oxidative stress, the oxidative stress index (OSI) was determined by calculating the ratio of the total peroxide to the total antioxidant potential. ${ }^{25}$

\section{Statistical analyses}

Data were analyzed in Statistical Package for Social Sciences 18.0 for Windows (SPSS Inc., Chicago, IL, USA). The Kolmogorov-Smirnov test was used to determine the distribution pattern of the data. The Kruskal-Wallis test was used to make comparisons among the five groups. Post hoc Mann-Whitney $U$-tests were used to determine which of the five groups differed from one another. Bonferroni correction was performed. All differences associated with a probability of 0.005 or less were considered statistically significant. Means and standard deviations were used to describe numerical variables.

\section{Results}

Results of the plasma analyses for HDL-chol, LDL-chol, TAG, Total-C, PON1 activity, TOS, TAS, OSI, and lipoprotein (a), as well as pretreatment and posttreatment body weight, are demonstrated in Table 1. Ovariectomy led to an increase in body weight compared with the sham operation $(P<0.001)$. In addition, oophorectomy led to a statistically significant $(P=0.002)$ increase in plasma Total-C levels compared with the sham operation. Compared with the sham group, the OVX group had no statistically significant differences in the HDL-chol, LDL-chol, TAG, TOS, TAS, OSI, or PON1 activity $(P>0.005)$. The E2-treated group had significantly elevated levels of LDL-chol, Total-C, TOS, and OSI in comparison with the control group $(P<0.005)$. Compared with the control group, the E2/Prog- and Gentreated groups had no statistically significant differences in HDL-chol, LDL-chol, Total-C, TAG, TAS, TOS, OSI, PON1 activity, or in pretreatment and posttreatment body weight $(P>0.005)$.

LDL-chol significantly decreased in the E2/Prog-treated group but not in the E2-treated group $(P=0.004)$. However, there was no significant difference in HDL-chol, Total-C, TAG, TAS, TOS, OSI, PON1 activity, or body weight $(P>0.005)$ between the given groups.

The E2-treated group had significantly elevated LDLchol and Total-C levels compared with the Gen-treated group $(P<0.005)$. However, there was no significant difference in HDL-chol, TAG, TAS, TOS, OSI, PON1 activity, 
Table I Biochemical data of study groups and statistical findings ( $P$-values) with regard to biochemical data

\begin{tabular}{|c|c|c|c|c|c|c|}
\hline $\begin{array}{l}\text { Group } \\
\text { parameters }\end{array}$ & $\begin{array}{l}\text { OVX+G } \\
(n=10)\end{array}$ & $\begin{array}{l}\text { OVX+E } \\
(n=10)\end{array}$ & $\begin{array}{l}\text { OVX+E/P } \\
(n=10)\end{array}$ & $\begin{array}{l}\text { Sham } \\
(n=10)\end{array}$ & $\begin{array}{l}\text { OVX } \\
(n=10)\end{array}$ & $P$-values \\
\hline HDL-chol (mg/dL) & $\begin{array}{l}25.7 \pm 2.0 \\
(22-29)\end{array}$ & $\begin{array}{l}26.2 \pm 2.6 \\
(21-30)\end{array}$ & $\begin{array}{l}26.8 \pm 2.2 \\
(23-30)\end{array}$ & $\begin{array}{l}23.9 \pm 11.2 \\
(18-556)\end{array}$ & $\begin{array}{l}27.6 \pm 11.6 \\
(15-59.2)\end{array}$ & 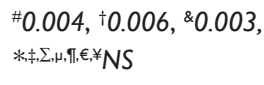 \\
\hline LDL-chol (mg/dL) & $\begin{array}{l}50.2 \pm 6.1 \\
(42.6-62.8)\end{array}$ & $\begin{array}{l}68.2 \pm 8.2 \\
(54-80)\end{array}$ & $\begin{array}{l}56.3 \pm 8.0 \\
(44.6-74.0)\end{array}$ & $\begin{array}{l}32.6 \pm 21.8 \\
(10.6-92.0)\end{array}$ & $\begin{array}{l}41.0 \pm 12.6 \\
(23.4-58.6)\end{array}$ & $\begin{array}{l}{ }^{8} 0.002,{ }^{\top} 0.001,{ }^{*} 0.004, \\
{ }^{\dagger} 0.002,{ }^{\Sigma} 0.000,{ }^{*} 0.002, \\
\mu, *, \neq, \epsilon \text { NS }\end{array}$ \\
\hline TAG (mg/dL) & $\begin{array}{l}42.7 \pm 9.7 \\
(29-57)\end{array}$ & $\begin{array}{l}42.9 \pm 6.2 \\
(35-58)\end{array}$ & $\begin{array}{l}38.3 \pm 14.1 \\
(20-59)\end{array}$ & $\begin{array}{l}62.1 \pm 13.6 \\
(46-92)\end{array}$ & $\begin{array}{l}56.6 \pm 18.1 \\
(36-103)\end{array}$ & $\begin{array}{l}{ }^{\#} 0.003,{ }^{8} 0.001,{ }^{8} 0.004, \\
\sharp, \pi, \epsilon, ¥, \Sigma, \mu, * N S\end{array}$ \\
\hline Total-C (mg/dL) & $\begin{array}{l}84.5 \pm 6.6 \\
(76-97)\end{array}$ & $\begin{array}{l}103.0 \pm 10.4 \\
(82-106)\end{array}$ & $\begin{array}{l}90.8 \pm 8.6 \\
(80-107)\end{array}$ & $\begin{array}{l}55.6 \pm 12.3 \\
(25-66)\end{array}$ & $\begin{array}{l}80.0 \pm 13.7 \\
(48-94)\end{array}$ & $\begin{array}{l}{ }^{\sharp} 0.000,{ }^{\top} 0.002, * 0.002, \\
{ }^{2} 0.000, \Sigma 0.001,{ }^{\dagger} 0.000, \\
{ }_{\neq}, \epsilon, \mu, * N S\end{array}$ \\
\hline $\begin{array}{l}\text { TAC ( } \mu \mathrm{M} \\
\text { Trolox equiv./L) }\end{array}$ & $\begin{array}{l}1.2 \pm 0.17 \\
(1.1-1.6)\end{array}$ & $\begin{array}{l}1.1 \pm 0.1 \\
(0.9-1.4)\end{array}$ & $\begin{array}{l}1.1 \pm 0.1 \\
(0.9-1.4)\end{array}$ & $\begin{array}{l}1.35 \pm 0.13 \\
(1.0-1.6)\end{array}$ & $\begin{array}{l}1.2 \pm 0.1 \\
(1.0-1.4)\end{array}$ & $*, \neq, \Sigma, \mu, \pi, \epsilon, \ldots, \#,+, \& N S$ \\
\hline $\begin{array}{l}\text { TOS ( } \mu \mathrm{M} \\
\mathrm{H}_{2} \mathrm{O}_{2} \text { equiv./L) }\end{array}$ & $\begin{array}{l}265.0 \pm|3| .2 \\
(50.9-448.6)\end{array}$ & $\begin{array}{l}226.0 \pm 97.2 \\
(81.1-426.6)\end{array}$ & $\begin{array}{l}\mid 89 \pm 106.1 \\
(36.5-439.0)\end{array}$ & $\begin{array}{l}94 . I \pm 68 . I \\
(35.5-236.6)\end{array}$ & $\begin{array}{l}105.4 \pm 53.6 \\
(45.3-189.4)\end{array}$ & $\begin{array}{l}{ }^{\#} 0.005,{ }^{2} 0.001,{ }^{\dagger} 0.003, \\
*, \neq,+, \pi, \epsilon, *, \& N S\end{array}$ \\
\hline PONI activity (U/L) & $\begin{array}{l}133.6 \pm 50.6 \\
(35.7-181.0)\end{array}$ & $\begin{array}{l}104.9 \pm 45.4 \\
(15.4-152.9)\end{array}$ & $\begin{array}{l}95.1 \pm 45.4 \\
(12.1-174.1)\end{array}$ & $\begin{array}{l}147.9 \pm 37.2 \\
(91.6-213.7)\end{array}$ & $\begin{array}{l}133.7 \pm 40.7 \\
(73.8-194.2)\end{array}$ & $*, \neq, \Sigma, \mu, \pi, \uparrow, \epsilon, \#, \#,+, \& N S$ \\
\hline OSI & $\begin{array}{l}204.5 \pm 101.3 \\
(43.9-347.7)\end{array}$ & $\begin{array}{l}198.6 \pm 92.4 \\
(7|.1-4| 4.1)\end{array}$ & $\begin{array}{l}164.2 \pm 101.9 \\
(30.1-4 \mid 8.1)\end{array}$ & $\begin{array}{l}68.1 \pm 46.5 \\
(27.5-164.3)\end{array}$ & $\begin{array}{l}85.2 \pm 45.7 \\
(39.4-180.1)\end{array}$ & 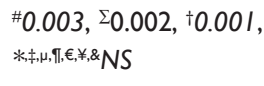 \\
\hline $\begin{array}{l}\text { Pre-t. body } \\
\text { weight (g) }\end{array}$ & $\begin{array}{l}262.1 \pm 14.7 \\
(254-278)\end{array}$ & $\begin{array}{l}263.1 \pm 10.4 \\
(250-28 I)\end{array}$ & $\begin{array}{l}262.4 \pm 9.3 \\
(25 I-280)\end{array}$ & $\begin{array}{l}262.4 \pm 14.7 \\
(250-287)\end{array}$ & $\begin{array}{l}261.6 \pm 9.6 \\
(251-280)\end{array}$ & $*, \pm, \Sigma, \mu, \uparrow, \uparrow, \ldots, \#, \#,+, \varepsilon N S$ \\
\hline $\begin{array}{l}\text { Post-t. body } \\
\text { weight (g) }\end{array}$ & $\begin{array}{l}317.3 \pm 36.9 \\
(263-370)\end{array}$ & $\begin{array}{l}340.8 \pm 40.4 \\
(26 I-39 I)\end{array}$ & $\begin{array}{l}325.9 \pm 13.2 \\
(305-352)\end{array}$ & $\begin{array}{l}260 \pm 15.3 \\
(243-289)\end{array}$ & $\begin{array}{l}305.8 \pm 25.3 \\
(256-334)\end{array}$ & $\begin{array}{l}* 0.001,{ }^{*} 0.001,{ }^{\dagger} 0.001, \\
{ }^{*} 0.000,{ }^{\ddagger}, \Sigma, \mu, \pi, \ldots \in \neq\end{array}$ \\
\hline Lipoprotein a & $<2$ & $<2$ & $<2$ & $<2$ & $<2$ & $*,+, \Sigma, \mu, \pi, \epsilon, \#, \#,+, \& N S$ \\
\hline
\end{tabular}

Notes: G: $10 \mathrm{mg} / \mathrm{kg} /$ day E: $0.014 \mathrm{mg} / \mathrm{kg} 17-\beta$ estradiol E/P: $0.014 \mathrm{mg} / \mathrm{kg} 17-\beta$ estradiol plus and $0.028 \mathrm{mg} / \mathrm{kg}$ drosperinone. Data are presented as mean \pm standart deviation (min-max). $P$-values denoting the outcomes of comparison of the parameters of biochemical five groups. $P<0.005$ was considered to be statistically significant. *Sham versus $O V X$, " $S$ ham versus $O V X+G$, ${ }^{+}$Sham versus $O V X+E$, ${ }^{8}$ Sham versus $O V X+E / P,{ }^{\ddagger} O V X$ versus $O V X+G,{ }^{\Sigma} O V X$ versus $O V X+E,{ }^{\mu} O V X$ versus $O V X+E / P,{ }^{\pi} O V X+G$ versus $O V X+E$, ${ }^{\epsilon} O V X+G$ versus $O V X+E / P,{ }^{*} O V X+E$ versus $O V X+E / P ; N S=$ Not significant between groups.

Abbreviations: HDL-chol, High-density lipoprotein; LDL-chol, Low-density lipoprotein; TAG, triacylglycerols; Total-C, Total cholesterol; TAC, Total antioxidant status; TOS, Total oxidant status; PONI activity, Paraoxonas activity; OSI, Oxidative stress index; Pre-t. body weight, Pretreatment body weight; Post-t. body weight, Posttreatment body weight; mg/dL, milligrams/deciliter; $\mu$ M, Micromol; $\mu$ M Trolox equiv./L, Mikromol Trolox equivalent per liter; $\mu M \mathrm{H}_{2} \mathrm{O}_{2}$ equiv./L, Mikromol hydrogen peroxide equivalent per liter; U/L, Units per liter; g, gram; OVX, ovariectomized.

or pretreatment and posttreatment body weight $(P>0.005)$ (Table 1) between the given groups. There was no statistically significant difference between the Gen- and E2/Prog-treated groups in biochemical parameters.

\section{Discussion}

In this study, no significant difference was found between the Gen-treated and control groups in HDL-chol, LDL-chol, TAG, Total-C, PON1, TAS, TOS, OSI, or pretreatment and posttreatment body weight. However, significantly lower levels of LDL-chol and Total-C were found in the Gen-treated group compared with the E2-treated group. In another study performed on 35 postmenopausal patients (20 Gen-treated and 15 placebo patients), lower levels of LDL-chol, Total-C, homocysteine, and visfatin were reported in the group of patients that were administered Gen compared with the control group. ${ }^{26}$ The difference might be explained by the fact that the present study was performed on rats. Furthermore, it can be stated that Gen does not lead to increased cardiovascular risk among postmenopausal women compared with other postmenopausal agents containing E2.

It has been shown that there is a shift to an atherogenic lipid and lipoprotein profile in the postmenopausal period. ${ }^{27}$ Furthermore, HDL-chol levels increase whereas LDL-chol levels decrease in amounts that exceed the effect due to the advanced age in the postmenopausal period. The distribution of central fat in women has been positively associated with the elevated levels of Total-C, TAG, and LDL-chol, and negatively associated with the reduced HDL-chol levels. ${ }^{28}$ In the present study, there was no difference in HDL-chol and LDL-chol levels, whereas Total-C levels and body weight were observed to increase after ovariectomy. Increased Total-C level and body weight are consistent with the findings of previous work reporting atherosclerosis tendency at menopause. ${ }^{29} 17-\beta$ E2 is a natural human estrogen and compensates for the physiological estrogen deficiency without affecting liver function. It reaches 
maximum plasma levels in 5-8 hours, and its half-life is approximately $12-14$ hours.

Menopausal hormone therapy (HRT) has been associated with beneficial effects on oxidative stress. Most studies favor its administration to menopausal women. Some studies, however, are not in favor of its use for treatment of menopausal symptoms due to the antioxidant and prooxidant characteristics of estrogen, in addition to several side effects it might have. It has also been suggested that MHT has no significant effects on oxidative stress levels. ${ }^{31,32}$ In the present study, no significant difference was found in TAS, TOS, or OSI levels after ovariectomy. Comparison between the Gen- and E2/ Prog-treated groups revealed that there was no significant difference between the groups in TAS, TOS, or OSI levels. On the other hand, the E2-treated group was observed to have elevated TOS levels. In the study of McManus et al the results do not support the role of estrogen as an antioxidant in vivo. ${ }^{33}$ There may be some explanations for the contradicting results in vivo and in vitro. Firstly, estrogen concentration in vitro is 104- to 106-fold higher compared with the concentration that is present in postmenopausal women who receive HRT (in vivo). ${ }^{32}$ In addition, estrogen impacts on LDL-chol particle size, causing increased proportion of tiny dense LDL-chol particles. In the end, these particles cause increased susceptibility to oxidation. ${ }^{34}$

In their study Evsen et al found reduced TOS and OSI levels and elevated TAS levels in the brain tissue of OVX rats treated with Gen, estrogen, or estrogen plus Prog. ${ }^{35}$ To the contrary, in the present study, the OVX rats treated with estrogen or Gen had significantly higher TOS and OSI levels compared with the control group. Effects of Gen depend on the ER- $\alpha / E R-\beta$ ratio for oxidative stress regulation and mitochondrial functionality. The effects on oxidative stress and mitochondria could be attributed, at least partially, to a higher ER- $\beta$ presence but could also be attributed to upregulation of ER- $\beta$ caused by Gen. ${ }^{36}$

Gen is likely to inhibit the formation of blood clots that are a major cause of heart attacks, as suggested by very early in vitro research. ${ }^{37}$ In the present study as well, the Gentreated group had significantly lower LDL-chol and Total-C levels compared with the E2-treated group. In the Heart and Estrogen/Progesterone Replacement Study (HERS), ${ }^{38}$ coronary artery disease (CAD)-related events were found to increase in the 1st year in the hormone (E2/Prog) group, while they were observed to decrease from the 3rd year to the 5 th year. However, this decrease did not continue in the subsequent years. The study lasted 6.8 years, hormone therapy did not reduce the mortality associated with atherosclerosis in women with CAD. ${ }^{38}$ The HERS concluded that it was not appropriate to use HRT to reduce the mortality associated with CAD in menopausal women.

In the Estrogen Replacement and Atherosclerosis (ERA) Study, ${ }^{39}$ LDL-chol levels were reported to decrease in the equine estrogen- and combined equine estrogen plus medroxyprogesterone acetate-treated groups $(9.4 \% \pm 20.9 \%$ and $16.5 \% \pm 21.8 \%$ ) compared with the placebo group $(1.3 \% \pm 21.5 \%)(P<0.02$ and $P<0.001$, respectively). No difference was observed in the occurrence rates of cardiovascular events in any of the treatment groups. No statistical difference was found in the minimal coronary artery diameter variations between the groups. The ERA study concluded that neither estrogen alone nor estrogen plus medroxyprogesterone acetate was effective to stop the progression of atherosclerosis in women with CAD. It was reported that estrogen replacement therapy should not be used in case of CAD with the expectation of cardiovascular benefit. ${ }^{39}$ In the present study, no difference was found in the lipoprotein levels between the Gen- and E2/Prog-treated group, while LDL-chol, Total-C, TOS, and OSI levels were significant higher in the E2-treated group compared with the control group.

In one of its studies, the World Health Institute (WHI) reported that osteoporosis decreased from 15 to ten in 10,000 person-years, colorectal carcinoma decreased from 16 to ten per 10,000, stroke-related heart attack increased from 30 to 37 in 10,000 person-years, breast cancer increased from 30 to 38 in 10,000 person-years, stroke increased from 21 to 29 in 10,000 person-years, and deep vein thrombosis (DVT) increased from 16 to 34 per 1000 person-years, both with combined HRT and estrogen replacement therapy in comparison with the placebo. ${ }^{40}$

In the present study, no difference was found in lipid profile and oxidative stress markers between the Gen-treated and control groups. On the other hand, the Gen-treated group had lower LDL-chol and Total-C levels compared with the E2-treated group. This is consistent with the increased heart attack and DVT rates found in HRT in the WHI study.

The antioxidative effects of Gen were investigated in many studies. Kim et al explored the effects of soybeans on lipid mechanisms and oxidative stress markers in rats fed with high-fat diet and found that soybeans were antihyperlipidemic and antioxidative. ${ }^{41}$ In the present study, no difference was found in HDL, LDL, TAG, TAS, TOS, OSI, PON1, Total-C, or pretreatment and posttreatment body weight between the Gen-treated and control groups. However, higher levels of LDL-chol and Total-C were observed in the Gen-treated group compared with the E2-treated group. In another study, one of the OVX rat groups received no treatment, while one group received Gen and another received E2. The study found 
that the antioxidative activity was higher in the Gen-treated group compared with the E2-treated and untreated groups. ${ }^{42}$ In the present study, no difference was found in the oxidative activity between the untreated and Gen-treated groups. However, the untreated group had significantly lower levels of TOS and OSI compared with the E2-treated group.

In human studies conducted with HRT, the effects of estrogen replacement therapy and E2/Prog therapy on serum TAG, Total-C, LDL-chol, and HDL-chol levels were investigated. Loh et al randomly selected 96 healthy postmenopausal women and administered $1 \mathrm{mg}$ E2 plus $0.5 \mathrm{mg}$ norethisterone acetate (NETA) and $2 \mathrm{mg}$ E2 plus $1 \mathrm{mg}$ NETA for 6 months in order to assess the effects of the low-dose E2 and NETA combination on lipid profile, bleeding profile, and menopausal symptoms. ${ }^{43}$ Total-C, LDL-chol, and TAG levels decreased in both treatment groups. In the present study, the OVX rats were administered $0.014 \mathrm{mg} / \mathrm{kg} 17-\beta \mathrm{E} 2$ plus $0.028 \mathrm{mg} / \mathrm{kg}$ drospirenone, $0.014 \mathrm{mg} / \mathrm{kg} 17-\beta \mathrm{E} 2$, or $10 \mathrm{mg} / \mathrm{kg}$ Gen. The doses administered in this study were consistent with those administered in other rat studies that used Gen in OVX rats. ${ }^{44,45}$

In the present study, Total-C, LDL-chol, and TAG levels did not decrease in any of the three treatment groups. Sporrong et al randomized 60 postmenopausal women into four groups and gave the patients $2 \mathrm{mg} 17 \beta \mathrm{E} 2$ plus NETA $1 \mathrm{mg}$ or $0.5 \mathrm{mg}$; or $2 \mathrm{mg} 17 \beta \mathrm{E} 2$ plus $5 \mathrm{mg}$ or $2.5 \mathrm{mg}$ megestrol acetate. They measured Total-C, LDL-chol, very low-density lipoprotein (VLDL-chol), and HDL-chol levels at months 1, 4, and 12, and found that the dose of progestin rather than its type was effective on the lipid metabolism. Furthermore, patients that were administered NETA did not demonstrate any changes in their lipids. ${ }^{46}$ In the present study, the comparison between the Gen-, E2/Prog-, and E2-treated groups showed that the Gen- and E2/Prog-treated groups had significantly lower LDL-chol levels compared with the E2-treated group.

In their study, Lee et $\mathrm{al}^{47}$ found that body weight and Total-C, TAG, and LDL-chol levels increased in the OVX rats, while HDL-chol levels decreased. These effects were reduced by exercise and/or soy isoflavone supplementation. The group of OVX rats treated with an isoflavone diet and exercise training had higher PON1 and Nitric oxide (NO) activities compared with the group of OVX rats treated with general diet. These results suggest that exercise combined with a soy isoflavone diet controls lipid profiles and the related enzyme, PON1, as well as NO activity and apoptosis in the aorta of OVX rats and thus has protective effects against cardiovascular risk factors. ${ }^{47}$ Similarly, in the present study, body weight increased in OVX rats, while no difference was found in the PON1 activity. Posttreatment body weight did not change and Total-C levels were observed to increase in OVX rats.
In conclusion, it was found in the present study that the Total-C levels and body weight increased in OVX rats, and HRTs did not have any significant effects on oxidative stress and lipid metabolites. However, the Gentreated group had significantly lower posttreatment levels of LDL-chol and Total-C compared with the E2-treated group. In this respect, it could be concluded that Gen therapy might be selected as the first-line option for the treatment of menopausal symptoms among women at risk for cardiovascular diseases that might occur in the postmenopausal period.

\section{Acknowledgment}

The authors are grateful to the Department of Obstetrics and Gynecology, Dicle University, Diyarbakir, Turkey, for their valuable support.

\section{Disclosure}

The authors report no conflicts of interest in this work.

\section{References}

1. Scott Jr, Dishan PJ, Hammond CB, Spellacy WN, editors. Danforth Obstetrics and Gynecology. Istanbul: the Great Books; 1986. English.

2. Santell RC, Chang YC, Nair MG, Helferich WG. Dietary genistein exerts estrogenic effects upon the uterus, mammary gland and the hypothalamic/ pituitary axis in rats. $J$ Nutr. 1997;127(2):263-229.

3. Speroff L, Whitcomb RW, Kempfert NJ, Boyd RA, Paulissen JB, Rowan JP. Efficacy and local tolerance of a low-dose, 7-day matrix estradiol transdermal system in the treatment of menopausal vasomotor symptoms. Obstetrics \& Gynecology. 1996;88(4 Pt 1):587-592.

4. Polfus LM, Gibbs RA, Boerwinkle E. Coronary heart disease and genetic variants with low phospholipase A2 activity. $N$ Engl $J$ Med. 2015;372(3):295-296.

5. Gök H. editor. Clinical Cardiology. İssue 1, Istanbul: Nobel Bookstore; 1996:97-171.

6. Wang Z, Chandrasena ER, Yuan Y, et al. Redox cycling of catechol estrogens generating apurinic/apyrimidinic sites and 8-oxo-deoxyguanosine via reactive oxygen species differentiates equine and human estrogens. Chem Res Toxicol. 2010;23(8):1365-1373.

7. Signorelli SS, Neri S, Sciacchitano S, et al. Behaviour of some indicators of oxidative stress in postmenopausal and fertile women. Maturitas. 2006;53(1):77-82

8. McLean RR. Proinflammatory cytokines and osteoporosis. Curr Osteoporos Rep. 2009;7(4):134-139.

9. King RJ, Whitehead MI. Assessment of the potency of orally administered progestins in women. Fertil Steril. 1986;46(6):1062-1066.

10. Krattenmacher R. Drospirenone: pharmacology and pharmacokinetics of a unique progestogen. Contraception. 2000;62(1):29-38.

11. EBSCO Complementary and Alternative Medicine (CAM). A Review of the Literature According to the National Center for Complementary and Alternative Medicine. The journal of supportive oncology. 2013;11(3): $1-23$.

12. Squadrito F, Altavilla D, Crisafulli A, et al. Effect of genistein on endothelial function in postmenopausal women: a randomized, doubleblind, controlled study. Am J Med. 2003;114(6):470-476.

13. Fanti O, Faugere MC, Gang Z, et al. Systematic administration of genistein partially prevents bone loss in ovariectomized rats in a nonestrogen-like mechanism [abstract]. Am J Clin Nutr. 1998;68(Suppl): S1517-S1518. 
14. Atteritano M, Marini H, Minutoli L, et al. Effects of the phytoestrogen genistein on some predictors of cardiovascular risk in osteopenic, postmenopausal women: a two-year randomized, double-blind, placebocontrolled study. J Clin Endocrinol Metab. 2007;92(8):3068-3075.

15. Cheng K, Chen KM, Ge BF, Zhen P, Gao YH, Ma HP. [Comparison research with icariin and genistein by anti-inflammatory reaction and angiogenesis pathway to inhibit bone loss on ovariectomized rats]. Zhong Yao Cai. 2014;37(4):627-631. Chinese.

16. Taylor CK, Levy RM, Elliott JC, Burnett BP. The effect of genistein aglycone on cancer and cancer risk: a review of in vitro, preclinical, and clinical studies. Nutr Rev. 2009;67(7):398-415.

17. Trieu VN, Uckun FM. Genistein is neuroprotective in murine models of familial amyotrophic lateral sclerosis and stroke. Biochem Biophys Res Commun. 1999;258(3):685-688.

18. Morán J, Garrido P, Cabello E, Alonso A, González C. Effects of estradiol and genistein on the insulin signaling pathway in the cerebral cortex of aged female rats. Exp Gerontol. 2014;58:104-112.

19. Mueller SO, Korach KS. Mechanisms of estrogen receptor-mediated agonistic and antagonistic effects. In: Metzler M, editor. Endocrine Disruptors Part: 1. Berlin: Springer-Verlag; 2001:1-26.

20. Diel P, Geis RB, Caldarelli A, et al. The differential ability of the phytoestrogen genistein and of estradiol to induce uterine weight and proliferation in the rat is associated with a substance specific modulation of uterine gene expression. Mol Cell Endocrinol. 2004;221(1-2):21-32.

21. Huang J, Nasr M, Kim Y, Matthews HR. Genistein inhibits protein histidine kinase. J Biol Chem. 1992;267(22):15511-15515.

22. Erel O. A novel automated method to measure total antioxidant response against potent free radical reactions. Clin Biochem. 2004;37(2): $112-119$.

23. Erel O. A new automated colorimetric method for measuring total oxidant status. Clin Biochem. 2005;38(12):1103-1111.

24. Eckerson HW, Romson J, Wyte C, La Du BN. The human serum paraoxonase polymorphism: identification of phenotypes by their response to salts. Am J Hum Genet. 1983;35(2):214-227.

25. Uzar E, Tamam Y, Evliyaoglu O, et al. Serum prolidase activity and oxidative status in patients with diabetic neuropathy. Neurol Sci. 2012; 33(4):875-880.

26. Irace $\mathrm{C}$, Marini $\mathrm{H}$, Bitto $\mathrm{A}$, et al. Genistein and endothelial function in postmenopausal women with metabolic syndrome. Eur J Clin Invest. 2013;43(10):1025-1031.

27. Sinem Beden. Effect of postmenopausal hormone treatment on serum lipid profile and coagulation factors. Journal of Experimental and Clinical Medicine. 2010:27(3).

28. del Ghianda S, Tonacchera M, Vitti P. Thyroid and menopause. Climacteric. 2014;17(3):225-234.

29. Whayne TF, Mukherjee D. Women, the menopause, hormone replacement therapy and coronary heart disease. Curr Opin Cardiol.2015 Epub Feb 18.

30. Sejal B. Doshi and Ashok Agarwal. The role of oxidative stress in menopause. J Midlife Health. 2013;4(3):140-146.

31. Bednarek-Tupikowska G, Tworowska U, Jedrychowska I, et al. Effects of oestradiol and oestroprogestin on erythrocyte antioxidative enzyme system activity in postmenopausal women. Clin Endocrinol (Oxf). 2006;64(4):463-468.

32. Clarkson TB, Meléndez GC, Appt SE. Timing hypothesis for postmenopausal hormone therapy: its origin, current status, and future. Menopause. 2013;20(3):342-353.

Drug Design, Development and Therapy

\section{Publish your work in this journal}

Drug Design, Development and Therapy is an international, peerreviewed open-access journal that spans the spectrum of drug design and development through to clinical applications. Clinical outcomes, patient safety, and programs for the development and effective, safe, and sustained use of medicines are a feature of the journal, which
33. McManus J, McEneny J, Young IS, Thompson W. The effect of various estrogens and progestagens on the susceptibility of LDL cholesterol to oxidation: P-66. Menopause. 1995;2(4):275-276.

34. Tribble DL, Holl LG, Wood PD, Krauss RM. Variations in oxidative susceptibility among six low density lipoprotein subfractions of differing density and particle size. Atherosclerosis. 1992;93(3):189-199.

35. Evsen MS, Ozler A, Gocmez C, et al. Effects of estrogen, estrogen/ progesteron combination and genistein treatments on oxidant/antioxidant status in the brain of ovariectomized rats. Eur Rev Med Pharmacol Sci. 2013;17(14):1869-1873.

36. Nadal-Serrano M, Pons DG, Sastre-Serra J, Blanquer-Rosselló Mdel M, Roca P, Oliver J. Genistein modulates oxidative stress in breast cancer cell lines according to $E R \alpha / E R \beta$ ratio: effects on mitochondrial functionality, sirtuins, uncoupling protein 2 and antioxidant enzymes. Int $J$ Biochem Cell Biol. 2013;45(9):2045-2051.

37. Tham DM, Gardner CD, Haskell WL. Clinical review 97: Potential health benefits of dietary phytoestrogens: a review of the clinical, epidemiological, and mechanistic evidence. J Clin Endocrinol Metab. 1998;83(7):2223-2235.

38. Grady D, Herrington D, Bittner V, et al; HERS Research Group. Cardiovascular disease outcomes during 6.8 years of hormone therapy. heart and estrogen/progestin replacement study follow-up (HERS II). JAMA. 2002;288(1):49-57.

39. Herrington DM, Reboussin DM, Brosnihan KB, et al. Effects of estrogen replacement on the progression of coronary atherosclerosis. $N$ Eng $J$ Med. 2000;343(8):522-529.

40. Rossouw JE, Anderson GL, Prentice RL, et al; Writing Group for the Women's Health Initiative Investigators. Risks and benefits of estrogen plus progestin in healthy postmenopausal women: principal results From the Women's Health Initiative randomized controlled trial. JAMA. 2002;288(3):321-333.

41. Kim DI, Kim KS, Kang JH, Kim HJ. Effect of Phellinus baumiibiotransformed soybean powder on lipid metabolism in rats. Prev Nutr Food Sci. 2013;18(2):98-103.

42. Squadrito F, Altavilla D, Squadrito G, et al. Genistein supplementation and estrogen replacement therapy improve endothelial dysfunction induced by ovariectomy in rats. Cardiovasc Res. 2000; 45(2):454-462.

43. Loh FH, Chen LH, Yu SL, Jorgensen LN. The efficacy of two dosages of a continuous combined hormone replacement regimen. Maturitas. 2002;41(2):123-131.

44. Manjanatha MG, Shelton SD, Rhodes BS et al. 17 Beta-estradiol and not genistein modulates lacI mutant frequency and types of mutation induced in the heart of ovariectomized big blue rats treated with 7 , 12-dimethylbenz[a] anthracene. OVX+rats+17-B2+E2+genistein "Environmental and molecular mutagenesis." Environ Mol Mutagen. 2005;45(1):70-79.

45. Morán J, Garrido P, Alonso A, Cabello E, González C. 17ß-Estradiol and genistein acute treatments improve some cerebral cortex homeostasis aspects deteriorated by aging in female rats. Exp Gerontol. 2013; 48(4):414-421.

46. Sporrong T, Hellgren M, Samsioe G, Mattsson LA. Metabolic effects of continuous estradiol-progestin therapy in postmenopausal women. Obstet Gynecol. 1989;73(5 Pt 1):754-758.

47. Lee J, Cho HS, Kim DY, et al. Combined effects of exercise and soy isoflavone diet on paraoxonase, nitric oxide and aortic apoptosis in ovariectomized rats. Appetite. 2012;58(2):462-469.

\section{Dovepress}

has also been accepted for indexing on PubMed Central. The manuscript management system is completely online and includes a very quick and fair peer-review system, which is all easy to use. Visit http://www.dovepress.com/testimonials.php to read real quotes from published authors. 